\title{
Multiple solutions for a quasilinear Schrödinger-Poisson system
}

Jing Zhang 1* $^{*}$

\author{
"Correspondence: \\ jinshizhangjing@163.com \\ ${ }^{1}$ Mathematics Sciences College, \\ Inner Mongolia Normal University, \\ Hohhot, 010022, P.R. China
}

\begin{abstract}
In this article, we consider the following quasilinear Schrödinger-Poisson system$$
\left\{\begin{array}{l}
-\Delta u+V(x) u-u \Delta\left(u^{2}\right)+K(x) \phi(x) u=g(x, u), \quad x \in \mathbb{R}^{3}, \\
-\Delta \phi=K(x) u^{2}, \quad x \in \mathbb{R}^{3},
\end{array}\right.
$$

where $V, K: \mathbb{R}^{3} \rightarrow \mathbb{R}$ and $g: \mathbb{R}^{3} \times \mathbb{R} \rightarrow \mathbb{R}$ are continuous functions; $g$ is of subcritical growth and has some monotonicity properties. The purpose of this paper is to find the ground state solution of (0.1), i.e., a nontrivial solution with the least possible energy by taking advantage of the generalized Nehari manifold approach, which was proposed by Szulkin and Weth. Furthermore, infinitely many geometrically distinct solutions are gained while $g$ is odd in $u$.
\end{abstract}

MSC: 35A15; 35J10; 35B09

Keywords: Generalized Nehari manifold; Quasilinear system; Schrödinger-Poisson; Multiple solutions

\section{Introduction}

In this article, we consider the following quasilinear Schrödinger-Poisson system:

$$
\left\{\begin{array}{l}
-\Delta u+V(x) u-u \Delta\left(u^{2}\right)+K(x) \phi(x) u=g(x, u), \quad x \in \mathbb{R}^{3} \\
-\Delta \phi=K(x) u^{2}, \quad x \in \mathbb{R}^{3}
\end{array}\right.
$$

where $V, K: \mathbb{R}^{3} \rightarrow \mathbb{R}$ and $g: \mathbb{R}^{3} \times \mathbb{R} \rightarrow \mathbb{R}$ are continuous functions.

The quasilinear Schrödinger-Poisson system had been introduced in [4, 20], which is a quantum mechanical model of extremely small devices in semiconductor nanostructures taking into account the quantum structure and the longitudinal field oscillations during the beam propagation.

The Schrödinger-Poisson system can be written as follows:

$$
\left\{\begin{array}{l}
-\Delta u+V(x) u+K(x) \phi(x) u=g(x, u), \quad x \in \mathbb{R}^{3}, \\
-\Delta \phi=K(x) u^{2}, \quad x \in \mathbb{R}^{3} .
\end{array}\right.
$$

(c) The Author(s) 2021. This article is licensed under a Creative Commons Attribution 4.0 International License, which permits use sharing, adaptation, distribution and reproduction in any medium or format, as long as you give appropriate credit to the original author(s) and the source, provide a link to the Creative Commons licence, and indicate if changes were made. The images or other third party material in this article are included in the article's Creative Commons licence, unless indicated otherwise in a credit line to the material. If material is not included in the article's Creative Commons licence and your intended use is not permitted by statutory regulation or exceeds the permitted use, you will need to obtain permission directly from the copyright holder. To view a copy of this licence, visit http://creativecommons.org/licenses/by/4.0/. 
There were many different growth conditions on the nonlinearity $g(x, u)$, such as subcritical growth [6, 21, 31, 33, 36], or the critical exponent growth [32, 34]. Moreover, many meaningful results have been obtained. For example, radial solutions $[8,11,22]$, ground state solutions [2, 3], and also semiclassical solutions [12, 18, 19].

The quasilinear Schrödinger equation

$$
-\Delta u+V(x) u-u \Delta\left(u^{2}\right)=g(x, u), \quad x \in \mathbb{R}^{N},
$$

has been accepted as a model of several physical phenomena in [23], and for the results about this quasilinear equation the reader can be referred to $[1,5,9,10,15,30]$.

At present, there are relatively few existing results about system (1.1), so it could be of interest to pay close attention to our discussion. When $K(x)=1$ in system (1.1), the authors of [7] took into account the system together with a 4-Laplacian operator, hence the existence of sign-changing solution with precisely two nodal domains was derived by using the approximation technique. When the system is considered with asymptotically linear $f(t)$ with respect to $t$ at infinity, the existence and asymptotic behavior of the ground state were studied in [13]. Figueiredo and Siciliano [16,17] paid close attention to two different systems with parameter $\varepsilon$ and critical growth, and obtained the existence of solutions for the former system in $\mathbb{R}^{3}$. They also proved asymptotic behavior of solutions whenever $\varepsilon \rightarrow 0$. Similar results were also achieved for the latter system in a bounded domain $\Omega$ in $\mathbb{R}^{2}$. The authors in [24] considered a system with radial potentials and discontinuous nonlinearity, and then obtained the multiplicity results of radial solutions by nonsmooth critical point theory. By utilizing Ekeland's variational principle, the authors of [25] obtained the existence of the ground state solution by seeking the solution of a new system, which is equivalent to system (1.1). By applying the mountain pass theorem and the concentrationcompactness principle, the existence of a solution to problem (1.1) was established in [35] when the asymptotic periodicity of potentials $V, K$ and nonlinearity $g$ were considered. To the best of our knowledge, no one made use of the generalized manifold method to show the existence of solutions of this quasilinear Schrödinger-Poisson system. On the basis of the existing results, our aim is to study the existence of a ground state solution and of infinitely many solutions.

Setting $G(x, u)=\int_{0}^{u} g(x, s) d s$, we suppose that $V, K$ and $g$ satisfy the following assumptions:

( $V) V$ is continuous, 1-periodic in $x_{i}, 1 \leq i \leq N$, and $V_{0}=\inf _{x \in \mathbb{R}^{3}} V(x)>0$;

(K) $K \in L^{2}\left(\mathbb{R}^{3}\right) \cap L^{\infty}\left(\mathbb{R}^{3}\right), \lim _{|x| \rightarrow \infty} K(x)=0, K(x) \geq 0$ for all $x \in \mathbb{R}^{3}, K \not \equiv 0$.

$\left(g_{1}\right) g \in C\left(\mathbb{R}^{3} \times \mathbb{R}, \mathbb{R}\right)$ is 1 -periodic in $x_{i}, 1 \leq i \leq N,|g(x, u)| \leq a\left(1+|u|^{p-1}\right)$ for some $a>0$ and $4<p<12$;

$\left(g_{2}\right) g(x, u)=o(u)$ uniformly in $x$ as $u \rightarrow 0$;

$\left(g_{3}\right) u \mapsto \frac{g(x, u)}{u^{3}}$ is positive for $u \neq 0$, nonincreasing on $(-\infty, 0)$, and nondecreasing on $(0,+\infty)$

$\left(g_{4}\right) \frac{G(x, u)}{u^{4}} \rightarrow \infty$ uniformly in $x$ as $|u| \rightarrow \infty$.

Let $*$ denote the action of $\mathbb{Z}^{3}$ on $H^{1}\left(\mathbb{R}^{3}\right)$ given by

$$
(k * u):=u(x-k), \quad k \in \mathbb{Z}^{3} .
$$


We note that if $u_{0}$ is a solution of (1.1), then so are all elements of the orbit of $\left(u_{0}, \phi_{0}\right)$ under the action of $\mathbb{Z}^{3}$, and the so-called orbit of $\left(u_{0}, \phi_{0}\right)$ is denoted as

$$
\mathcal{O}\left(u_{0}, \phi_{0}\right):=\left\{u_{0}(\cdot-k), \phi_{0}(\cdot-k): k \in \mathbb{Z}^{3}\right\}
$$

Two solutions $\left(u_{1}, \phi_{1}\right)$ and $\left(u_{2}, \phi_{2}\right)$ of (1.1) are said to be geometrically distinct if $\mathcal{O}\left(u_{1}, \phi_{1}\right)$ and $\mathcal{O}\left(u_{2}, \phi_{2}\right)$ are disjoint.

Now we state our main results.

Theorem 1.1 Suppose $(V),(K)$, and $\left(g_{1}\right)-\left(g_{4}\right)$ hold. Then the problem (1.1) has a ground state solution.

Theorem 1.2 Suppose $(V),(K)$, and $\left(g_{1}\right)-\left(g_{4}\right)$ hold, and $g$ is odd in $u$. Then the problem (1.1) admits infinitely many geometrically distinct solutions.

\section{Preliminary results}

In this section, we introduce the variational framework associated with problem (1.1). It follows from $(V)$ that we can define a new norm

$$
\|u\|=\left(\int_{\mathbb{R}^{3}}|\nabla u|^{2}+V(x) u^{2}\right)^{\frac{1}{2}}
$$

which is equivalent to the usual norm of $H^{1}\left(\mathbb{R}^{3}\right)$. The usual norm in the Lebesgue space $L^{p}\left(\mathbb{R}^{3}\right)$ is denoted by $|u|_{p}$. Then there exists $r_{p}>0$ such $|u|_{p} \leq r_{p}\|u\|$ for $p \in[1,6]$. Also $\|u\|_{D^{1,2}}=\int_{\mathbb{R}^{3}}\left(|\nabla u|^{2}\right)^{1 / 2}$ denotes the norm of $D^{1,2}\left(\mathbb{R}^{3}\right)$. It is more convenient for our purposes than the standard one and will be used henceforth. For a functional $F$, we put

$$
F^{b}:=\left\{u \in H^{1}\left(\mathbb{R}^{3}\right): F(u) \leq b\right\}, \quad F_{a}:=\left\{u \in H^{1}\left(\mathbb{R}^{3}\right): F(u) \geq a\right\}, \quad F_{a}^{b}:=F_{a} \cap F^{b} .
$$

As far as we know, system (1.1) can be easily transformed into a single nonlinear Schrödinger equation with a nonlocal term. Briefly, the Poisson equation is solved by using the Lax-Milgram theorem, so for all $u \in H^{1}\left(\mathbb{R}^{3}\right)$, a unique $\phi_{u} \in D^{1,2}\left(\mathbb{R}^{3}\right)$ is gained, such that $-\Delta \phi=K(x) u^{2}$ and, when inserted into the first equation, it gives

$$
-\Delta u+V(x) u-u \Delta\left(u^{2}\right)+K(x) \phi_{u} u=g(x, u) .
$$

Actually, for each $u \in H^{1}\left(\mathbb{R}^{3}\right)$, we define an operator $T_{u}$ on $D^{1,2}\left(\mathbb{R}^{3}\right)$ by

$$
T_{u}(v)=\int_{\mathbb{R}^{3}} K(x) u^{2} v d x .
$$

Hölder inequality and the fact $K \in L^{2}\left(\mathbb{R}^{3}\right)$ yield that there is a constant $C>0$ such that for every $v \in D^{1,2}\left(\mathbb{R}^{3}\right)$,

$$
\left|T_{u}(v)\right| \leq C|K|_{2}\|u\|^{2}\|v\|_{D^{1,2}\left(\mathbb{R}^{3}\right)} .
$$

Hence, by the Riesz representation theorem, there exists a unique $\phi_{u} \in D^{1,2}\left(\mathbb{R}^{3}\right)$ such that

$$
\int_{\mathbb{R}^{3}} \nabla \phi_{u} \nabla v d x=\int_{\mathbb{R}^{3}} K(x) u^{2} v d x
$$


Thus $\phi_{u}$ is a weak solution of $-\Delta \phi_{u}=K(x) u^{2}$ and can be represented by

$$
\phi_{u}(x)=\frac{1}{4 \pi} \int_{\mathbb{R}^{3}} \frac{K(y)}{|x-y|} u^{2}(y) d y .
$$

Moreover, it is obvious that

$$
\left\|\phi_{u}\right\|_{D^{1,2}\left(\mathbb{R}^{3}\right)}=\left\|T_{u}\right\|_{\mathcal{L}\left(D^{1,2}, \mathbb{R}\right)} \leq C|K|_{2}\|u\|^{2} .
$$

Also there is

$$
\int_{\mathbb{R}^{3}} K(x) \phi_{u}(x) u^{2} d x \leq C|K|_{2}\|u\|^{2}\|\phi\|_{D^{1,2}\left(\mathbb{R}^{3}\right)} \leq C|K|_{2}^{2}\|u\|^{4}
$$

Moreover,

$$
\begin{aligned}
\left|\phi_{u}(x)\right| & =\frac{1}{4 \pi}\left|\int_{B_{1}(0)} \frac{K(y)}{|x-y|} u^{2}(y) d y+\int_{B_{1}^{c}(0)} \frac{K(y)}{|x-y|} u^{2}(y) d y\right| \\
& \leq \frac{1}{4 \pi}\left(|K|_{\infty}\left(\int_{B_{1}(0)} \frac{1}{|x-y|^{2}} d y\right)^{\frac{1}{2}}|u|_{4}^{2}+|K|_{4}\left(\int_{B_{1}^{c}(0)} \frac{1}{|x-y|^{4}} d y\right)^{\frac{1}{4}}|u|_{4}^{2}\right) \\
& <C(K)|u|_{4}^{2} .
\end{aligned}
$$

Then

$$
\int_{\mathbb{R}^{3}} K(x) \phi_{u} u^{2} d x \leq\left|\phi_{u}\right|_{\infty}|K|_{2}|u|_{4}^{2} \leq C(K)|K|_{2}|u|_{4}^{4}
$$

Let us now define the operator $\Phi: H^{1}\left(\mathbb{R}^{3}\right) \rightarrow D^{1,2}\left(\mathbb{R}^{3}\right)$ as

$$
\Phi[u]=\phi_{u}
$$

then $\Phi$ has the following properties [11]:

\section{Lemma 2.1}

(1) $\Phi$ is continuous and maps bounded sets into bounded sets;

(2) $\Phi[u] \geq 0$;

(3) If $u_{n} \rightarrow u$ in $H^{1}\left(\mathbb{R}^{3}\right)$, then $\Phi\left[u_{n}\right] \rightarrow \Phi[u]$ in $D^{1,2}\left(\mathbb{R}^{3}\right)$;

(4) $\Phi[t u]=t^{2} \Phi[u]$ for all $t \in \mathbb{R}$.

We observe that (1.1) is formally the Euler-Lagrange equation associated with the energy functional

$$
\begin{aligned}
J(u)= & \frac{1}{2} \int_{\mathbb{R}^{3}}\left(1+2 u^{2}\right)|\nabla u|^{2} d x+\frac{1}{2} \int_{\mathbb{R}^{3}} V(x) u^{2} d x \\
& +\frac{1}{4} \int_{\mathbb{R}^{3}} K(x) \phi_{u} u^{2} d x-\int_{\mathbb{R}^{3}} G(x, u) d x .
\end{aligned}
$$

From the variational point of view, the first difficulty associated with the problem (1.1) is finding an appropriate function space where the functional $J$ is well defined. In order 
to avoid the difficulty which is caused by the quasilinear term, we take advantage of the change of variable introduced by [23], that is, we consider $v=f^{-1}(u)$, where $f$ is defined by

$$
\begin{aligned}
& f^{\prime}(t)=\frac{1}{\left(1+2 f^{2}(t)\right)^{\frac{1}{2}}} \quad \text { on }[0,+\infty), \\
& f(t)=-f(-t) \quad \text { on }(-\infty, 0],
\end{aligned}
$$

having the following properties, which have been proved in $[9,14]$.

Lemma 2.2 The function $f$ satisfies the following properties:

(1) $f$ is uniquely defined, $C^{\infty}$, and invertible;

(2) $\left|f^{\prime}(t)\right| \leq 1$ for all $t \in \mathbb{R}$;

(3) $|f(t)| \leq|t|$ for all $t \in \mathbb{R}$;

(4) $f(t) / t \rightarrow 1$ as $t \rightarrow 0$;

(5) $f(t) / \sqrt{t} \rightarrow 2^{1 / 4}$ as $t \rightarrow+\infty$;

(6) $f(t) / 2 \leq t f^{\prime}(t) \leq f(t)$ for all $t \geq 0$;

(7) $|f(t)| \leq 2^{1 / 4}|t|^{1 / 2}$ for all $t \in \mathbb{R}$;

(8) $f^{2}(t) / 2 \leq f(t) f^{\prime}(t) t \leq f^{2}(t)$ for all $t \in \mathbb{R}$;

(9) There exists a positive constant $C$ such that

$$
|f(t)| \geq \begin{cases}C|t|, & |t| \leq 1 \\ C|t|^{1 / 2}, & |t| \geq 1\end{cases}
$$

(10) $\left|f(t) f^{\prime}(t)\right| \leq 1 / \sqrt{2}$ for all $t \in \mathbb{R}$;

(11) The function $f(t) f^{\prime}(t) t^{-1}$ is strictly decreasing for all $t>0$;

(12) The function $f^{p}(t) f^{\prime}(t) t^{-1}$ is strictly increasing for all $p \geq 3$ and $t>0$.

So, after the change of variables, from $J$ we obtain the following functional:

$$
\begin{aligned}
I(v)= & \frac{1}{2} \int_{\mathbb{R}^{3}}|\nabla v|^{2} d x+\frac{1}{2} \int_{\mathbb{R}^{3}} V(x) f^{2}(v) d x \\
& +\frac{1}{4} \int_{\mathbb{R}^{3}} K(x) \phi_{f(v)} f^{2}(v) d x-\int_{\mathbb{R}^{3}} G(x, f(v)) d x,
\end{aligned}
$$

which is well defined in $H^{1}\left(\mathbb{R}^{3}\right)$ and belongs to $C^{1}$ under the hypotheses $(V),(K),\left(g_{1}\right)$, and $\left(g_{2}\right)$. Moreover, the critical points of $I$ are the weak solutions of the problem

$$
-\Delta v+V(x) f(v) f^{\prime}(v)+K(x) \phi_{f(v)} f(v) f^{\prime}(v)=g(x, f(v)) f^{\prime}(v), \quad v \in H^{1}\left(\mathbb{R}^{3}\right),
$$

that is,

$$
\begin{aligned}
\left\langle I^{\prime}(v), w\right\rangle= & \int_{\mathbb{R}^{3}}\left(\nabla v \nabla w+V(x) f(v) f^{\prime}(v) w\right) d x \\
& +\int_{\mathbb{R}^{3}} K(x) \phi_{f(v)} f(v) f^{\prime}(v) w d x-\int_{\mathbb{R}^{3}} g(x, f(v)) f^{\prime}(v) w d x=0,
\end{aligned}
$$

for all $v, w \in H^{1}\left(\mathbb{R}^{3}\right)$. It has been shown in [9] that if $v \in H^{1}\left(\mathbb{R}^{3}\right)$ is a critical point of the functional $I$, then $u=f(v) \in H^{1}\left(\mathbb{R}^{3}\right)$ and $u$ is a solution of (1.1). 
We also observe that, in order to obtain a nonnegative solution for $(1.1)$, we set $g(x, s)=$ 0 for all $x \in \mathbb{R}^{3}, s<0$. Indeed, let $v$ be a critical point of $I$. Taking $w=-v^{-}$, where $v^{-}=$ $\max \{-v, 0\}$, we get

$$
\int_{\mathbb{R}^{3}}\left(\left|\nabla v^{-}\right|^{2}+V(x) f(v) f^{\prime}(v)\left(-v^{-}\right)\right) d x=0 .
$$

Since $f(v)\left(-v^{-}\right) \geq 0$, we have

$$
\int_{\mathbb{R}^{3}}\left|\nabla v^{-}\right|^{2} d x=0 \quad \text { and } \quad \int_{\mathbb{R}^{3}} \frac{V(x) f(v)\left(-v^{-}\right)}{\sqrt{1+2 f^{2}(v)}} d x=0 .
$$

Hence we may conclude that $v^{-}=0$ almost everywhere in $\mathbb{R}^{3}$ and, therefore, $v=v^{+} \geq 0$. As $u=f(v)$, we conclude that $u$ is a nonnegative solution for the problem (1.1).

Let

$$
M=\left\{v \in H^{1}\left(\mathbb{R}^{3}\right) \backslash\{0\}:\left\langle I^{\prime}(v), v\right\rangle=0\right\} .
$$

Recall that $M$ is called the Nehari manifold. We do not know whether $M$ is of class $C^{1}$ under our assumptions and therefore we cannot use the minimax theory directly on $M$. To overcome this difficulty, we employ an argument developed in [27, 28].

\section{Proof of the main results}

We assume that $(V),(K)$, and $\left(g_{1}\right)-\left(g_{4}\right)$ are satisfied from now on. Firstly, $\left(g_{1}\right)$ and $\left(g_{2}\right)$ imply that for each $\varepsilon>0$ there is $C_{\varepsilon}>0$ such that

$$
|g(x, u)| \leq \varepsilon|u|+C_{\varepsilon}|u|^{p-1}, \quad \text { for all } u \in \mathbb{R}
$$

By using $\left(g_{2}\right)$ and $\left(g_{3}\right)$, one can easily check that

$$
G(x, u) \geq 0 \quad \text { and } \quad g(x, u) u \geq 4 G(x, u)>0 \quad \text { if } u \neq 0
$$

For $t>0$, let

$$
\begin{aligned}
h(t) & =I(t u) \\
& =\frac{t^{2}}{2} \int_{\mathbb{R}^{3}}|\nabla u|^{2}+\frac{1}{2} \int_{\mathbb{R}^{3}} V(x) f^{2}(t u)+\frac{1}{4} \int_{\mathbb{R}^{3}} K(x) \phi_{f(t u)} f^{2}(t u)-\int_{\mathbb{R}^{3}} G(x, f(t u)) .
\end{aligned}
$$

Lemma 3.1 For each $u \in H^{1}\left(\mathbb{R}^{3}\right) \backslash\{0\}$, there exists a unique $t_{u}=t(u)>0$ such that $m(u):=$ $t_{u} u \in M$ and $I(m(u))=\max _{t \in \mathbb{R}^{+}} I(t u)$.

Proof By (3.1) and Lemma 2.2(7), for $\varepsilon$ sufficiently small,

$$
\begin{aligned}
h(t) & \geq \frac{t^{2}}{2} \int_{\mathbb{R}^{3}}|\nabla u|^{2}+\frac{1}{2} \int_{\mathbb{R}^{3}} V(x) f^{2}(t u)-\frac{\varepsilon}{2} \int_{\mathbb{R}^{3}} f^{2}(t u)-\frac{C_{\varepsilon}}{p} \int_{\mathbb{R}^{3}}|f(t u)|^{p} \\
& \geq \frac{t^{2}}{2} \int_{\mathbb{R}^{3}}|\nabla u|^{2}-C_{1} t^{\frac{p}{2}} \int_{\mathbb{R}^{3}}|u|^{\frac{p}{2}}
\end{aligned}
$$

since $p>4$ and $u \neq 0$, so $h(t)>0$ whenever $t>0$ is small enough. 
Using Lemma 2.2, (3) and (7), and (2.2),

$$
\begin{aligned}
h(t) \leq & \frac{t^{2}}{2} \int_{\mathbb{R}^{3}}|\nabla u|^{2}+\frac{t^{2}}{2} \int_{\mathbb{R}^{3}} V(x) u^{2}+\frac{C(K)|K|_{2}}{4} \int_{\mathbb{R}^{3}}|f(t u)|^{4}-\int_{\mathbb{R}^{3}} G(x, f(t u)) \\
\leq & \frac{t^{2}}{2} \int_{\mathbb{R}^{3}}|\nabla u|^{2}+\frac{t^{2}}{2} \int_{\mathbb{R}^{3}} V(x) u^{2}+\frac{C(K)|K|_{2}}{2} t^{2} \int_{\mathbb{R}^{3}} u^{2} \\
& -t^{2} \int_{\mathbb{R}^{3}} \frac{G(x, f(t u))}{f^{4}(t u)} \cdot \frac{f^{4}(t u)}{(t u)^{2}} \cdot u^{2},
\end{aligned}
$$

then we can easily gain that $h(t) \rightarrow-\infty$ as $t \rightarrow \infty$ according to (2.1), Lemma 2.2(5), $\left(g_{4}\right)$, and Fatou's lemma. Therefore, $\max _{t>0} h(t)$ is achieved at some $t_{u}=t(u)>0$, so that $h^{\prime}\left(t_{u}\right)=$ 0 and then $t_{u} u \in M$.

It remains to show the uniqueness of $t_{u}$. Suppose by contradiction that there exists a $t_{1}>0$ with $t_{u} \neq t_{1}$ such that $h^{\prime}\left(t_{1}\right)=I^{\prime}\left(t_{1} u\right)=J^{\prime}\left(t_{1} v\right)=0$, then we have

$$
\frac{\|v\|^{2}}{\left(t_{1}\right)^{2}}+4 \int_{\mathbb{R}^{3}} v^{2}|\nabla v|^{2}+\int_{\mathbb{R}^{3}} K(x) \phi_{\nu} v^{2}=\int_{\mathbb{R}^{3}} \frac{g\left(x, t_{1} v\right)}{\left(t_{1} v\right)^{3}} v^{4},
$$

where $u=f^{-1}(v)$. Together with

$$
\frac{\|v\|^{2}}{\left(t_{u}\right)^{2}}+4 \int_{\mathbb{R}^{3}} v^{2}|\nabla v|^{2}+\int_{\mathbb{R}^{3}} K(x) \phi_{v} v^{2}=\int_{\mathbb{R}^{3}} \frac{g\left(x, t_{u} v\right)}{\left(t_{u} v\right)^{3}} v^{4},
$$

this implies that

$$
\left(\frac{1}{\left(t_{1}\right)^{2}}-\frac{1}{\left(t_{u}\right)^{2}}\right)\|v\|^{2}=\left(\frac{g\left(x, t_{1} v\right)}{\left(t_{1} v\right)^{3}}-\frac{g\left(x, t_{u} v\right)}{\left(t_{u} v\right)^{3}}\right) v^{4},
$$

contrary to $\left(g_{3}\right)$.

\section{Lemma 3.2}

(1) There exists $\rho>0$ such that $c=\inf _{M} I \geq \inf _{S_{\rho}} I>0$, where $S_{\rho}=\left\{u \in H^{1}\left(\mathbb{R}^{3}\right):\|u\|=\rho\right\}$.

(2) There exists $\alpha_{0}>0$ such that $\|u\|^{2} \geq \alpha_{0}$ for all $u \in M$.

(3) I is coercive on $M$, i.e., $I(u) \rightarrow \infty$ as $\|u\| \rightarrow \infty, u \in M$.

Proof (1) According to [15], $\int_{\mathbb{R}^{3}}|\nabla u|^{2}+\int_{\mathbb{R}^{3}} V(x) f^{2}(u) \geq C\|u\|^{2}$ whenever $\|u\| \leq \rho$. By (3.1) and Lemma 2.2, (3) and (7), we have

$$
\begin{aligned}
\int_{\mathbb{R}^{3}} G(x, f(u)) & \leq \frac{\varepsilon}{2} \int_{\mathbb{R}^{3}} f^{2}(u)+\frac{C_{\varepsilon}}{p} \int_{\mathbb{R}^{3}}|f(u)|^{p} \\
& \leq \frac{\varepsilon}{2} \int_{\mathbb{R}^{3}}|u|^{2}+\frac{C_{2} C_{\varepsilon}}{p} \int_{\mathbb{R}^{3}}|u|^{\frac{p}{2}} \leq C_{3} \varepsilon\|u\|^{2}+C_{4} C_{\varepsilon}\|u\|^{\frac{p}{2}},
\end{aligned}
$$

for sufficiently small $\varepsilon$,

$$
I(u) \geq C_{5}\|u\|^{2}-C_{6}\|u\|^{\frac{p}{2}},
$$


and then $\inf _{S_{\rho}} I>0$ is obtained when $\rho$ is small enough. The inequality $\inf _{M} I \geq \inf _{S_{\rho}} I$ is a consequence of Lemma 3.1 since for every $u \in M$ there is $s>0$ such that $s u \in S_{\rho}$ and $I\left(t_{u} u\right) \geq I(s u)$.

(2) For $u \in M$, by Lemma 2.2(3) and (2.1), we have

$$
\begin{aligned}
0 & <c \leq I(u)=\frac{1}{2} \int_{\mathbb{R}^{3}}|\nabla u|^{2}+\frac{1}{2} \int_{\mathbb{R}^{3}} V(x) u^{2}++\frac{1}{4} \int_{\mathbb{R}^{3}} K(x) \phi_{u} u^{2}-\int_{\mathbb{R}^{3}} G(x, f(u)) \\
& \leq \frac{1}{2} \int_{\mathbb{R}^{3}}|\nabla u|^{2}+\frac{1}{2} \int_{\mathbb{R}^{3}} V(x) u^{2}+\frac{1}{4} \int_{\mathbb{R}^{3}} K(x) \phi_{u} u^{2} \leq \frac{1}{2}\|u\|^{2}+C|K|_{2}^{2}\|u\|^{4},
\end{aligned}
$$

which means that there exists $\alpha_{0}>0$ such that $\|u\|^{2} \geq \alpha_{0}$.

(3) Arguing indirectly, let $\left(u_{n}\right) \subset M$ be a sequence such that $\left\|u_{n}\right\| \rightarrow \infty$ and $I\left(u_{n}\right) \leq d$ for some $d$. Set $v_{n}=u_{n} /\left\|u_{n}\right\|$. Then $\left\|v_{n}\right\|=1$ and, passing to a subsequence, $v_{n} \rightarrow v$ in $H^{1}\left(\mathbb{R}^{3}\right)$ and $v_{n} \rightarrow v$ a.e. in $\mathbb{R}^{3}$. Suppose

$$
\lim _{n \rightarrow \infty} \max _{y \in \mathbb{R}^{3}} \int_{B_{1}(y)} v_{n}^{2}=0,
$$

where $B_{1}(x)$ is the ball in $\mathbb{R}^{3}$ with center $x$ and radius 1 , then it follows that $v_{n} \rightarrow 0$ in $L^{r}\left(\mathbb{R}^{3}\right)$ for $2<r<6$ by Lions' lemma (cf. [29, Lemma 1.21]). Using (3.1) and Lemma 2.2, (3) and (7), we see that $G\left(x, f\left(s v_{n}\right)\right) \rightarrow 0$ for all $s \in \mathbb{R}$. Hence by Lemma 2.2(9),

$$
\begin{aligned}
d & \geq I\left(u_{n}\right) \geq I\left(s v_{n}\right) \\
& =\frac{s^{2}}{2} \int_{\mathbb{R}^{3}}\left|\nabla v_{n}\right|^{2}+\frac{1}{2} \int_{\mathbb{R}^{3}} V(x) f^{2}\left(s v_{n}\right)+\frac{1}{4} \int_{\mathbb{R}^{3}} K(x) \phi_{f\left(s v_{n}\right)} f^{2}\left(s v_{n}\right)-\int_{\mathbb{R}^{3}} G\left(x, f\left(s v_{n}\right)\right) \\
& \geq \frac{s^{2}}{2} \int_{\mathbb{R}^{3}}\left|\nabla v_{n}\right|^{2}+\frac{C^{2} s^{2}}{2} \int_{\left|s v_{n}\right| \leq 1} V(x) v_{n}^{2}-\int_{\mathbb{R}^{3}} G\left(x, f\left(s v_{n}\right)\right) \\
& =\frac{s^{2}}{2} \int_{\mathbb{R}^{3}}\left|\nabla v_{n}\right|^{2}+\frac{C^{2} s^{2}}{2} \int_{\mathbb{R}^{3}} V(x) v_{n}^{2}-\frac{C^{2}}{2} \int_{\left|s v_{n}\right| \geq 1} V(x)\left(s v_{n}\right)^{2}-\int_{\mathbb{R}^{3}} G\left(x, f\left(s v_{n}\right)\right) \\
& \geq \frac{s^{2}}{2} \min \left\{1, C^{2}\right\}-C_{7} \int_{\mathbb{R}^{3}}\left(s v_{n}\right)^{p / 2}-\int_{\mathbb{R}^{3}} G\left(x, f\left(s v_{n}\right)\right) \\
& \rightarrow \frac{s^{2}}{2} \min \left\{1, C^{2}\right\} .
\end{aligned}
$$

This yields a contradiction if we choose a sufficiently large $s$. Hence (3.3) is not true and, since $I$ and $M$ are invariant under the action of $\mathbb{Z}^{3}$, after a suitable $\mathbb{Z}^{3}$ translation it follows that $v_{n} \rightarrow v \neq 0$ in $L_{\text {loc }}^{2}\left(\mathbb{Z}^{3}\right)$. Since $\left|u_{n}(x)\right| \rightarrow \infty$ if $v(x) \neq 0$, it follows from Lemma 2.2(5), $\left(g_{4}\right)$, and Fatou's lemma that

$$
\int_{\mathbb{R}^{3}} \frac{G\left(x, f\left(u_{n}\right)\right)}{\left\|u_{n}\right\|^{2}}=\int_{\mathbb{R}^{3}} \frac{G\left(x, f\left(u_{n}\right)\right)}{f^{4}\left(u_{n}\right)} \cdot \frac{f^{4}\left(u_{n}\right)}{u_{n}^{2}} \cdot v_{n}^{2} \rightarrow \infty,
$$

and also, by Lemma 2.2, (3) and (7), as well as (2.2),

$$
0 \leq \frac{I\left(u_{n}\right)}{\left\|u_{n}\right\|^{2}} \leq \frac{1}{2}+\frac{C(K)|K|_{2}\left|u_{n}\right|_{2}^{2}}{2\left\|u_{n}\right\|^{2}}-\int_{\mathbb{R}^{3}} \frac{G\left(x, f\left(u_{n}\right)\right)}{\left\|u_{n}\right\|^{2}} \rightarrow-\infty,
$$

thus the proof is completed. 
Lemma 3.3 If $\mathcal{V}$ is a compact subset of $H^{1}\left(\mathbb{R}^{3}\right) \backslash\{0\}$, then there exists $R>0$ such that $I \leq 0$ on $\mathbb{R}^{+} \mathcal{V} \backslash B_{R}(0)$.

Proof We may assume without loss of generality that $\mathcal{V} \subset S$. Arguing by contradiction, suppose there exist $u_{n} \in \mathcal{V}$ and $w_{n}=m\left(u_{n}\right)=t_{n} u_{n}$ such that $I\left(w_{n}\right) \geq 0$ and $t_{n} \rightarrow \infty$ as $n \rightarrow \infty$. Passing to a subsequence, there is $u \in H^{1}\left(\mathbb{R}^{3}\right)$ with $\|u\|=1$ such that $u_{n} \rightarrow u \in S$. Since $\left|w_{n}(x)\right| \rightarrow \infty$ if $u(x) \neq 0$, then by (2.2) and Lemma 2.2(7) it follows that

$$
\frac{\int_{\mathbb{R}^{3}} K(x) \phi_{f\left(w_{n}\right.} f^{2}\left(w_{n}\right)}{\left\|w_{n}\right\|^{2}} \leq \frac{C(K)|K|_{2}\left|f\left(w_{n}\right)\right|_{4}^{4}}{\left\|w_{n}\right\|^{2}} \leq \frac{2 C(K)|K|_{2}\left|w_{n}\right|_{2}^{2}}{\left\|w_{n}\right\|^{2}}
$$

so by $\left(g_{4}\right)$, Lemma 2.2(5), and Fatou's lemma we get that

$$
\int_{\mathbb{R}^{3}} \frac{G\left(x, f\left(w_{n}\right)\right)}{\left\|w_{n}\right\|^{2}}=\int_{\mathbb{R}^{3}} \frac{G\left(x, f\left(w_{n}\right)\right) u_{n}^{2}}{w_{n}^{2}}=\int_{\mathbb{R}^{3}} \frac{G\left(x, f\left(w_{n}\right)\right)}{f^{4}\left(w_{n}\right)} \cdot \frac{f^{4}\left(w_{n}\right)}{w_{n}^{2}} \cdot u_{n}^{2} \rightarrow \infty .
$$

Therefore, by Lemma 2.2(3),

$$
0 \leq \frac{I\left(w_{n}\right)}{\left\|w_{n}\right\|^{2}} \leq \frac{1}{2}+\frac{C(K)|K|_{2}\left|w_{n}\right|_{2}^{2}}{2\left\|w_{n}\right\|^{2}}-\int_{\mathbb{R}^{3}} \frac{G\left(x, f\left(w_{n}\right)\right)}{\left\|w_{n}\right\|^{2}} \rightarrow-\infty,
$$

a contradiction.

Recall that $S$ is the unit sphere in $H^{1}\left(\mathbb{R}^{3}\right)$ and define the mapping $m: S \rightarrow M$ by setting

$$
m(w):=t_{w} w,
$$

where $t_{w}$ is as in Lemma 3.1. Note that $\|m(w)\|=t_{w}$. Lemmas 3.4 and 3.5 below are taken from [28] (see Proposition 8 and Corollary 10 there). That the hypotheses in [28] are satisfied is a consequence of Lemmas 3.1, 3.2 and 3.3 above. Indeed, if $h(t)=I(t w)$ and $w \in S$, then $h^{\prime}(t)>0$ for $0<t<t_{w}$ and $h^{\prime}(t)<0$ for $t>t_{w}$ by Lemma 3.1, $t_{w} \geq \delta>0$ by Lemma 3.2, and $t_{w} \leq R$ for $w \in \mathcal{V} \subset S$ by Lemma 3.3.

Lemma 3.4 The mapping $m$ is continuous. Moreover, the mapping $m$ is a homeomorphism between $S$ and $M$, and the inverse of $m$ is given by $m^{-1}(u)=\frac{u}{\|u\|}$.

We shall consider the functional $\Psi: S \rightarrow \mathbb{R}$ given by

$$
\Psi(w)=I(m(w)) .
$$

\section{Lemma 3.5}

(1) $\Psi \in C^{1}(S, \mathbb{R})$ and

$$
\left\langle\Psi^{\prime}(w), z\right\rangle=\|m(w)\|\left\langle I^{\prime}(m(w)), z\right\rangle \quad \text { for all } z \in T_{w}(S)=\left\{v \in H^{1}\left(\mathbb{R}^{3}\right),\langle v, w\rangle=0\right\} .
$$

(2) If $\left(w_{n}\right)$ is a Palais-Smale sequence for $\Psi$, then $\left(m\left(w_{n}\right)\right)$ is a Palais-Smale sequence for I. If $\left(u_{n}\right) \subset M$ is a bounded Palais-Smale sequence for $I$, then $\left(m^{-1}\left(u_{n}\right)\right)$ is a Palais-Smale sequence for $\Psi$. 
(3) $w$ is a critical point of $\Psi$ if and only if $m(w)$ is a nontrivial critical point of $I$.

Moreover, the corresponding values of $\Psi$ and $I$ coincide and $\inf _{S} \Psi=\inf _{M} I$.

(4) If I is even, then so is $\Psi$.

Lemma 3.6 If $u_{n} \rightarrow u$ in $H^{1}\left(\mathbb{R}^{3}\right)$, then

(1) $\int_{\mathbb{R}^{3}} K(x) \phi_{f\left(u_{n}\right)} f\left(u_{n}\right) f^{\prime}\left(u_{n}\right) u_{n} \rightarrow \int_{\mathbb{R}^{3}} K(x) \phi_{f(u)} f(u) f^{\prime}(u) u$;

(2) $\int_{\mathbb{R}^{3}} K(x) \phi_{f\left(u_{n}\right)} f^{2}\left(u_{n}\right) \rightarrow \int_{\mathbb{R}^{3}} K(x) \phi_{f(u)} f^{2}(u)$.

Proof (1) Because

$$
\begin{aligned}
& \int_{\mathbb{R}^{3}}\left|K(x) \phi_{f\left(u_{n}\right)} f\left(u_{n}\right) f^{\prime}\left(u_{n}\right) u_{n}-K(x) \phi_{f(u)} f(u) f^{\prime}(u) u\right| \\
& \leq|K|_{\infty} \int_{\mathbb{R}^{3}}\left|\phi_{f\left(u_{n}\right)} f\left(u_{n}\right) f^{\prime}\left(u_{n}\right) u_{n}-\phi_{f(u)} f(u) f^{\prime}(u) u\right| \\
& \leq|K|_{\infty}\left(\int_{\mathbb{R}^{3}}\left|\left(\phi_{f\left(u_{n}\right)}-\phi_{f(u)}\right) f\left(u_{n}\right) f^{\prime}\left(u_{n}\right) u_{n}\right|\right. \\
& \quad+\int_{\mathbb{R}^{3}}\left|\phi_{f(u)}\left[f\left(u_{n}\right) f^{\prime}\left(u_{n}\right) u_{n}-f(u) f^{\prime}\left(u_{n}\right) u_{n}\right]\right| \\
& \left.\quad+\int_{\mathbb{R}^{3}}\left|\phi_{f(u)}\left[f(u) f^{\prime}\left(u_{n}\right) u_{n}-f(u) f^{\prime}(u) u_{n}\right]\right|+\int_{\mathbb{R}^{3}}\left|\phi_{f(u)}\left[f(u) f^{\prime}(u) u_{n}-f(u) f^{\prime}(u) u\right]\right|\right) \\
& =|K|_{\infty}\left(I_{1}+I_{2}+I_{3}+I_{4}\right) .
\end{aligned}
$$

If $u_{n}$ is bounded in $H^{1}\left(\mathbb{R}^{3}\right)$, then passing to a subsequence gives that $u_{n} \rightarrow u$ in $H^{1}\left(\mathbb{R}^{3}\right)$ and $u_{n} \rightarrow u$ a.e. in $\mathbb{R}^{3}$. Then by (2.1) it follows that $\phi_{u_{n}}$ is bounded in $D^{1,2}\left(\mathbb{R}^{3}\right)$, and also $\phi_{u_{n}}$ is bounded in $L^{6}\left(\mathbb{R}^{3}\right)$. Then by Lemma $2.2(3)$ we obtain that $\phi_{f\left(u_{n}\right)}$ is bounded in $L^{6}\left(\mathbb{R}^{3}\right)$, so that $\phi_{f\left(u_{n}\right)}-\phi_{f\left(u_{n}\right)}$ is bounded in $L^{6}\left(\mathbb{R}^{3}\right)$. Suppose $\phi_{f\left(u_{n}\right)}-\phi_{f\left(u_{n}\right)} \rightarrow u_{0}$. Since $u_{n} \rightarrow u$ a.e. in $\mathbb{R}^{3}$ and due to the uniqueness of limit, we have $\phi_{f\left(u_{n}\right)} \rightarrow \phi_{f\left(u_{n}\right)}$ in $L^{6}\left(\mathbb{R}^{3}\right)$. Moreover, by Lemma 2.2, (8) and (3),

$$
\int_{\mathbb{R}^{3}}\left|f\left(u_{n}\right) f^{\prime}\left(u_{n}\right) u_{n}\right|^{\frac{6}{5}} \leq \int_{\mathbb{R}^{3}}\left|u_{n}\right|^{\frac{12}{5}} \leq C_{8}\left\|u_{n}\right\|^{\frac{12}{5}}
$$

so $f\left(u_{n}\right) f^{\prime}\left(u_{n}\right) u_{n} \in L^{\frac{6}{5}}\left(\mathbb{R}^{3}\right)$, and then $I_{1} \rightarrow 0$. Moreover, $I_{2} \rightarrow 0, I_{3} \rightarrow 0$, and $I_{4} \rightarrow 0$ can be obtained in a similar way.

(2) Observe that

$$
\begin{aligned}
& \int_{\mathbb{R}^{3}} K(x)\left|\phi_{f\left(u_{n}\right)} f^{2}\left(u_{n}\right)-\phi_{f(u)} f^{2}(u)\right| \\
& \quad \leq|K|_{\infty} \int_{\mathbb{R}^{3}}\left|\phi_{f\left(u_{n}\right)} f^{2}\left(u_{n}\right)-\phi_{f(u)} f^{2}(u)\right| \\
& \quad \leq|K|_{\infty}\left(\int_{\mathbb{R}^{3}}\left|\phi_{f\left(u_{n}\right)}-\phi_{f(u)}\right| f^{2}\left(u_{n}\right)+\int_{\mathbb{R}^{3}}\left|\phi_{f(u)}\left(f^{2}\left(u_{n}\right)-f^{2}(u)\right)\right|\right) \\
& \quad=|K|_{\infty}\left(I_{5}+I_{6}\right) .
\end{aligned}
$$

We also can use the same method, which was used in (1) to prove $I_{5} \rightarrow 0$ and $I_{6} \rightarrow 0$. And then the proof is completed. 
Now we are in a position to prove Theorem 1.1.

Proof of Theorem 1.1 We take advantage of an argument in [27]. Let $c=\inf _{M} I$. It follows from Lemma 3.2(1) that $c>0$. Moreover, if $u_{0} \in M$ satisfies $I\left(u_{0}\right)=c$, then $m^{-1}\left(u_{0}\right) \in S$ is a minimizer of $\Psi$ and therefore a critical point of $\Psi$, thus, by Lemma 3.5(3), $u_{0}$ is a critical point of $I$. It remains to show that there exists a minimizer $u$ of $\left.I\right|_{M}$. By Ekeland's variational principle [29], there exists a sequence $\left(w_{n}\right) \subset S$ with $\Psi\left(w_{n}\right) \rightarrow c$ and $\Psi^{\prime}\left(w_{n}\right) \rightarrow 0$ as $n \rightarrow \infty$. Set $u_{n}=m\left(w_{n}\right)$, then, from Lemma 3.5(2), we conclude that $I\left(u_{n}\right) \rightarrow c$ and $I^{\prime}\left(u_{n}\right) \rightarrow 0$ as $n \rightarrow \infty$. Obviously, $\left(u_{n}\right)$ is bounded by Lemma 3.2(3). Therefore $u_{n} \rightarrow u$ after passing to a subsequence. Suppose

$$
\lim _{n \rightarrow \infty} \sup _{y \in \mathbb{R}^{3}} \int_{B_{1}(y)} u_{n}^{2} d x=0,
$$

then by P.L. Lion's concentration compactness lemma (see [29, Lemma 1.21]) we get

$$
u_{n} \rightarrow 0 \quad \text { in } L^{r}\left(\mathbb{R}^{3}\right) \text { for } 2<r<6 .
$$

Furthermore, by Lemma 2.2, (6), (8) and (9),

$$
\begin{aligned}
o\left(\left\|u_{n}\right\|\right)= & \left\langle I^{\prime}\left(u_{n}\right), u_{n}\right\rangle \\
= & \int_{\mathbb{R}^{3}}\left|\nabla u_{n}\right|^{2}+\int_{\mathbb{R}^{3}} V(x) f\left(u_{n}\right) f^{\prime}\left(u_{n}\right) u_{n}+\int_{\mathbb{R}^{3}} K(x) \phi_{f\left(u_{n}\right)} f\left(u_{n}\right) f^{\prime}\left(u_{n}\right) u_{n} d x \\
& -\int_{\mathbb{R}^{3}} g\left(x, f\left(u_{n}\right)\right) f^{\prime}\left(u_{n}\right) u_{n} \\
\geq & \frac{1}{2} \int_{\mathbb{R}^{3}}\left|\nabla u_{n}\right|^{2}+\frac{1}{2} \int_{\mathbb{R}^{3}} V(x) f^{2}\left(u_{n}\right)+\frac{1}{2} \int_{\mathbb{R}^{3}} K(x) \phi_{f\left(u_{n}\right)} f^{2}\left(u_{n}\right) \\
& -\int_{\mathbb{R}^{3}} g\left(x, f\left(u_{n}\right)\right) f\left(u_{n}\right) \\
\geq & \frac{1}{2} \int_{\mathbb{R}^{3}}\left|\nabla u_{n}\right|^{2}+\frac{1}{2} \int_{\left|u_{n}\right| \leq 1} V(x) f^{2}\left(u_{n}\right)-\int_{\mathbb{R}^{3}} g\left(x, f\left(u_{n}\right)\right) f\left(u_{n}\right) \\
\geq & \frac{1}{2} \int_{\mathbb{R}^{3}}\left|\nabla u_{n}\right|^{2}+\frac{C^{2}}{2} \int_{\left|u_{n}\right| \leq 1} V(x) u_{n}^{2}-\int_{\mathbb{R}^{3}} g\left(x, f\left(u_{n}\right)\right) f\left(u_{n}\right) \\
= & \frac{1}{2} \int_{\mathbb{R}^{3}}\left|\nabla u_{n}\right|^{2}+\frac{C^{2}}{2} \int_{\mathbb{R}^{3}} V(x) u_{n}^{2}-\frac{C^{2}}{2} \int_{\left|u_{n}\right| \geq 1} V(x) u_{n}^{2}-\int_{\mathbb{R}^{3}} g\left(x, f\left(u_{n}\right)\right) f\left(u_{n}\right) \\
\geq & \frac{1}{2} \min \left\{1, C^{2}\right\}\left\|u_{n}\right\|^{2}-\frac{C^{2}}{2} \int_{\left|u_{n}\right| \geq 1} V(x) u_{n}^{2}-\int_{\mathbb{R}^{3}} g\left(x, f\left(u_{n}\right)\right) f\left(u_{n}\right) \\
\geq & \frac{1}{2} \min \left\{1, C^{2}\right\}\left\|u_{n}\right\|^{2}-C_{8} \int_{\mathbb{R}^{3}}\left(u_{n}\right)^{p / 2}-\int_{\mathbb{R}^{3}} g\left(x, f\left(u_{n}\right)\right) f\left(u_{n}\right) .
\end{aligned}
$$

According to (3.1) and using Lemma 2.2, (3) and (7), then

$$
g\left(x, f\left(u_{n}\right)\right) f\left(u_{n}\right) \leq \varepsilon\left|f\left(u_{n}\right)\right|^{2}+C_{\varepsilon}\left|f\left(u_{n}\right)\right|^{p} \leq \varepsilon\left|u_{n}\right|^{2}+2^{\frac{p}{4}} C_{\varepsilon}\left|u_{n}\right|^{\frac{p}{2}},
$$

which means that $\int_{\mathbb{R}^{3}} g\left(x, f\left(u_{n}\right)\right) f\left(u_{n}\right) \rightarrow 0$. Moreover,

$$
o\left(\left\|u_{n}\right\|\right)=\left\langle I^{\prime}\left(u_{n}\right), u_{n}\right\rangle \geq \frac{1}{2} \min \left\{1, C^{2}\right\}\left\|u_{n}\right\|^{2}-o\left(\left\|u_{n}\right\|\right),
$$


which yields a contradiction, so that (3.4) cannot hold. Thus, after a suitable $\mathbb{Z}^{3}$ translation, up to a subsequence we have $u_{n} \rightarrow u \neq 0$ and it is well known that $I^{\prime}(u)=0$. Accordingly, $u \in M$, and also that $I(u) \geq c$. In order to complete the proof, we only need to show that $I(u) \leq c$. Note that

$$
\begin{aligned}
c+ & o(1) \\
= & I\left(u_{n}\right)-\frac{1}{2}\left\langle I^{\prime}\left(u_{n}\right), u_{n}\right\rangle \\
= & \frac{1}{2} \int_{\mathbb{R}^{3}}\left|\nabla u_{n}\right|^{2}+\frac{1}{2} \int_{\mathbb{R}^{3}} V(x) f^{2}\left(u_{n}\right)+\frac{1}{4} \int_{\mathbb{R}^{3}} K(x) \phi_{f\left(u_{n}\right)} f^{2}\left(u_{n}\right)-\int_{\mathbb{R}^{3}} G\left(x, f\left(u_{n}\right)\right) \\
& -\left(\frac{1}{2} \int_{\mathbb{R}^{3}}\left|\nabla u_{n}\right|^{2}+\frac{1}{2} \int_{\mathbb{R}^{3}} V(x) f\left(u_{n}\right) f^{\prime}\left(u_{n}\right) u_{n}\right. \\
& \left.+\frac{1}{2} \int_{\mathbb{R}^{3}} K(x) \phi_{f\left(u_{n}\right)} f\left(u_{n}\right) f^{\prime}\left(u_{n}\right) u_{n}-\frac{1}{2} \int_{\mathbb{R}^{3}} g\left(x, f\left(u_{n}\right)\right) f^{\prime}\left(u_{n}\right) u_{n}\right) \\
= & \frac{1}{2} \int_{\mathbb{R}^{3}} V(x)\left(f^{2}\left(u_{n}\right)-f\left(u_{n}\right) f^{\prime}\left(u_{n}\right) u_{n}\right) \\
& +\left(\frac{1}{2} \int_{\mathbb{R}^{3}} g\left(x, f\left(u_{n}\right)\right) f^{\prime}\left(u_{n}\right) u_{n}-\int_{\mathbb{R}^{3}} G\left(x, f\left(u_{n}\right)\right)\right) \\
& +\left(\frac{1}{4} \int_{\mathbb{R}^{3}} K(x) \phi_{f\left(u_{n}\right)} f^{2}\left(u_{n}\right)-\frac{1}{2} \int_{\mathbb{R}^{3}} K(x) \phi_{f\left(u_{n}\right)} f\left(u_{n}\right) f^{\prime}\left(u_{n}\right) u_{n}\right) .
\end{aligned}
$$

From Lemma 2.2(8) and Fatou's lemma, we have

$$
\begin{aligned}
& \lim _{n \rightarrow \infty} \inf \int_{\mathbb{R}^{3}} V(x)\left(f^{2}\left(u_{n}\right)-f\left(u_{n}\right) f^{\prime}\left(u_{n}\right) u_{n}\right) \\
& \geq \int_{\mathbb{R}^{3}} \lim _{n \rightarrow \infty} \inf V(x)\left(f^{2}\left(u_{n}\right)-f\left(u_{n}\right) f^{\prime}\left(u_{n}\right) u_{n}\right) \\
& \quad=\int_{\mathbb{R}^{3}} V(x)\left(f^{2}(u)-f(u) f^{\prime}(u) u\right)
\end{aligned}
$$

and, by Lemma 2.2(6), (3.2), and Fatou's lemma,

$$
\begin{aligned}
& \lim _{n \rightarrow \infty} \inf \left(\frac{1}{2} \int_{\mathbb{R}^{3}} g\left(x, f\left(u_{n}\right)\right) f^{\prime}\left(u_{n}\right) u_{n}-\int_{\mathbb{R}^{3}} G\left(x, f\left(u_{n}\right)\right)\right) \\
& \geq \int_{\mathbb{R}^{3}} \lim _{n \rightarrow \infty} \inf \left(\frac{1}{2} g\left(x, f\left(u_{n}\right)\right) f^{\prime}\left(u_{n}\right) u_{n}-G\left(x, f\left(u_{n}\right)\right)\right) \\
& \quad=\frac{1}{2} \int_{\mathbb{R}^{3}} g(x, f(u)) f^{\prime}(u) u-\int_{\mathbb{R}^{3}} G(x, f(u)) .
\end{aligned}
$$

Therefore, by Lemma 3.6, we have

$$
c+o(1)=I\left(u_{n}\right)-\frac{1}{2}\left\langle I^{\prime}\left(u_{n}\right), u_{n}\right\rangle \geq I(u)-\frac{1}{2}\left\langle I^{\prime}(u), u\right\rangle=I(u)+o(1),
$$

which implies $I(u) \leq c$, thus Theorem 1.1 is proved.

In the following, we devote ourselves to the proof of the multiplicity result of Theorem 1.2. In addition to the assumptions $(V),(K)$, and $\left(g_{1}\right)-\left(g_{4}\right)$, we also suppose that $g$ is 
odd in $u$. Hereafter we use the following notation:

$$
H:=\left\{w \in S: \Psi^{\prime}(w)=0\right\}, \quad H_{d}:=\{w \in H: \Psi(w)=d\} .
$$

Denote by $\mathcal{F}:=H / \mathbb{Z}$ the set consisting of arbitrarily chosen representatives of the orbit of $H$. Since $\Psi$ is even, we may assume $\mathcal{F}=-\mathcal{F}$. As in Remark 2.12 of [27], since $m, m^{-1}$ are equivariant and $I, \Psi$ are invariant with respect to the action of $\mathbb{Z}^{3}$, it follows from Lemma 3.5(3) that the critical orbits $\mathcal{O}(u) \subset M$ of $I$ are in one-to-one correspondence with the critical orbits $\mathcal{O}(w) \subset S$ of $\Psi$. Hence the proof of Theorem 1.2 will be completed once we show that $\Psi$ has infinitely many critical orbits, i.e., the set $\mathcal{F}$ is infinite. Arguing indirectly, from now on we assume that

$\mathcal{F}$ is a finite set.

Lemma 3.7 The mapping $m^{-1}$ defined in Lemma 3.4 is Lipschitz continuous.

Proof We use an argument from [27]. By Lemma 3.2(2), we have, for all $u, v \in M$,

$$
\begin{aligned}
\left\|m^{-1}(u)-m^{-1}(v)\right\| & =\left\|\frac{u}{\|u\|}-\frac{v}{\|v\|}\right\| \\
& =\left\|\frac{u-v}{\|u\|}+\frac{(\|v\|-\|u\|) v}{\|u\|\|v\|}\right\| \\
& \leq \frac{2}{\|u\|}\|u-n\| \leq \frac{2}{\sqrt{\alpha_{0}}}\|u-n\| .
\end{aligned}
$$

According to [27], we shall show that Palais-Smale sequences have a certain discreteness property which yields some perfect properties of the corresponding pseudogradient flow (see Lemma 3.11 below). Firstly, we need some preparations involving $H$. The following result has been proved in Lemma 2.13 of [27]:

Lemma $3.8 k:=\inf \{\|v-w\|: v, w \in H, v \neq w\}>0$.

Lemma 3.9 If $\left\{u_{n}^{1}\right\},\left\{u_{n}^{2}\right\}$ are bounded in $H^{1}\left(\mathbb{R}^{3}\right)$, then

(1) there exists $C>0$, depending only on the bound on $\left\|u_{n}^{1}\right\|$ and $\left\|u_{n}^{2}\right\|$, such that

$$
\begin{gathered}
\int_{\mathbb{R}^{3}}\left|\nabla\left(u_{n}^{1}-u_{n}^{2}\right)\right|^{2}+\int_{\mathbb{R}^{3}} V(x)\left[f^{\prime}\left(u_{n}^{1}\right) f\left(u_{n}^{1}\right)-f^{\prime}\left(u_{n}^{2}\right) f\left(u_{n}^{2}\right)\right]\left(u_{n}^{1}-u_{n}^{2}\right) \\
\geq C \int_{\mathbb{R}^{3}}\left|\nabla\left(u_{n}^{1}-u_{n}^{2}\right)\right|^{2}+V(x)\left(u_{n}^{1}-u_{n}^{2}\right)^{2} \equiv C\left\|u_{n}^{1}-u_{n}^{2}\right\|^{2}
\end{gathered}
$$

(2) for each $\varepsilon>0$, there exists $C_{\varepsilon}>0$, depending only on the bound on $\left\|u_{n}^{1}\right\|$ and $\left\|u_{n}^{2}\right\|$, such that

$$
\left|\int_{\mathbb{R}^{3}} h_{n}(x)\left(u_{n}^{1}-u_{n}^{2}\right)\right| \leq \varepsilon\left\|u_{n}^{1}-u_{n}^{2}\right\|+C_{\varepsilon}\left|u_{n}^{1}-u_{n}^{2}\right|_{\frac{p}{2}},
$$

where

$$
h_{n}(x):=g\left(x, f\left(u_{n}^{1}\right)\right) f^{\prime}\left(u_{n}^{1}\right)-g\left(x, f\left(u_{n}^{2}\right)\right) f^{\prime}\left(u_{n}^{2}\right)=h_{n}^{1}(x)-h_{n}^{2}(x)
$$


(3) there exists $C(K)>0$, depending only on the bound on $\left\|u_{n}^{1}\right\|$ and $\left\|u_{n}^{2}\right\|$, such that

$$
\left|\int_{\mathbb{R}^{3}} K(x) \zeta_{n}(x)\left(u_{n}^{1}-u_{n}^{2}\right)\right| \leq C(K)\left|u_{n}^{1}-u_{n}^{2}\right|_{3}
$$

where

$$
\zeta_{n}(x)=\phi_{f\left(u_{n}^{1}\right)} f\left(u_{n}^{1}\right) f^{\prime}\left(u_{n}^{1}\right)-\phi_{f\left(u_{n}^{2}\right.} f\left(u_{n}^{2}\right) f^{\prime}\left(u_{n}^{2}\right) .
$$

Proof The proof of (1) and (2) can be found in [15], so we only prove (3). According to Lemma 2.2, (1) and (3),

$$
\begin{aligned}
& \left|\int_{\mathbb{R}^{3}} K(x) \zeta_{n}(x)\left(u_{n}^{1}-u_{n}^{2}\right)\right| \\
& \quad \leq|K|_{\infty}\left|\int_{\mathbb{R}^{3}}\left(\phi_{u_{n}^{1}} u_{n}^{1}+\phi_{u_{n}^{2}} u_{n}^{2}\right)\left(u_{n}^{1}-u_{n}^{2}\right)\right| \\
& \quad \leq|K|_{\infty}\left(\left|\phi_{u_{n}^{1}}\right|_{6}\left|u_{n}^{1}\right|_{2}\left|u_{n}^{1}-u_{n}^{2}\right|_{3}+\left|\phi_{u_{n}^{2}}\right|_{6}\left|u_{n}^{2}\right|_{2}\left|u_{n}^{1}-u_{n}^{2}\right|_{3}\right) \\
& \quad \leq C(K)\left|u_{n}^{1}-u_{n}^{2}\right|_{3} .
\end{aligned}
$$

Lemma 3.10 Let $d \geq c . I f\left(v_{n}^{1}\right),\left(v_{n}^{2}\right) \subset \Psi^{d}$ are two Palais-Smale sequences for $\Psi$, then either $\left\|v_{n}^{1}-v_{n}^{2}\right\| \rightarrow 0$ as $n \rightarrow \infty$ or $\lim _{\sup _{n \rightarrow \infty}}\left\|v_{n}^{1}-v_{n}^{2}\right\| \geq \rho(d)>0$, where $\rho(d)$ depends on $d$ but not on the particular choice of Palais-Smale sequences.

Proof Our argument is an adaptation of the proof of Lemma 2.14 in [27]. Let $u_{n}^{1}=m\left(v_{n}^{1}\right)$ and $u_{n}^{2}=m\left(v_{n}^{2}\right)$ for $n \in \mathbb{N}$. Then by Lemmas 3.5(2) and 3.2(3), both sequences $\left(u_{n}^{1}\right),\left(u_{n}^{2}\right) \subset$ $I^{d} \cap M$ are bounded Palais-Smale sequences for $I$. We will consider two cases: either $\left(u_{n}^{1}-\right.$ $\left.u_{n}^{2}\right)$ is vanishing, i.e., for each $r>0$,

$$
\lim _{n \rightarrow \infty} \max _{y \in \mathbb{R}^{3}} \int_{B_{r}(y)}\left|u_{n}^{1}-u_{n}^{2}\right|^{2}=0
$$

or nonvanishing, i.e., for each $r, \delta>0$ and any sequence $\left(y_{n}\right) \subset \mathbb{R}^{3}$, we have that

$$
\lim _{n \rightarrow \infty} \int_{B_{r}\left(y_{n}\right)}\left|u_{n}^{1}-u_{n}^{2}\right|^{2} \geq \delta>0
$$

Case 1: $\left(u_{n}^{1}-u_{n}^{2}\right)$ is vanishing. Then $u_{n}^{1}-u_{n}^{2} \rightarrow 0$ in $L^{r}\left(\mathbb{R}^{3}\right)$ for $r \in(2,6)$ by P.L. Lion's lemma (see [29, Lemma 1.21]). By Lemma 3.9,

$$
\begin{aligned}
C \| & u_{n}^{1}-u_{n}^{2} \|^{2} \\
\leq & \int_{\mathbb{R}^{3}}\left|\nabla\left(u_{n}^{1}-u_{n}^{2}\right)\right|^{2}+\int_{\mathbb{R}^{3}} V(x)\left[f^{\prime}\left(u_{n}^{1}\right) f\left(u_{n}^{1}\right)-f^{\prime}\left(u_{n}^{2}\right) f\left(u_{n}^{2}\right)\right]\left(u_{n}^{1}-u_{n}^{2}\right) \\
= & \left\langle I^{\prime}\left(u_{n}^{1}\right), u_{n}^{1}-u_{n}^{2}\right\rangle-\left\langle I^{\prime}\left(u_{n}^{2}\right), u_{n}^{1}-u_{n}^{2}\right\rangle \\
& -\int_{\mathbb{R}^{3}} K(x) \zeta_{n}(x)\left(u_{n}^{1}-u_{n}^{2}\right)+\int_{\mathbb{R}^{3}} h_{n}(x)\left(u_{n}^{1}-u_{n}^{2}\right) \\
\leq & C(K)\left|u_{n}^{1}-u_{n}^{2}\right|_{3}+2 \varepsilon\left\|u_{n}^{1}-u_{n}^{2}\right\|+C_{\varepsilon}\left|u_{n}^{1}-u_{n}^{2}\right|_{\frac{p}{2}}
\end{aligned}
$$


where $C, C(K), C_{\varepsilon}$ do not depend on the choice of $\varepsilon$. Therefore, $\lim _{\sup _{n \rightarrow \infty}}\left\|u_{n}^{1}-u_{n}^{2}\right\|^{2} \leq$ $\limsup _{n \rightarrow \infty} 2 \varepsilon\left\|u_{n}^{1}-u_{n}^{2}\right\|$ for each $\varepsilon>0$, and so that $\left\|u_{n}^{1}-u_{n}^{2}\right\| \rightarrow 0$. As a consequence of Lemma 3.7, $m^{-1}$ is Lipschitz continuous so that $\left\|v_{n}^{1}-v_{n}^{2}\right\|=\left\|m^{-1}\left(u_{n}^{1}\right)-m^{-1}\left(u_{n}^{2}\right)\right\| \rightarrow 0$ as $n \rightarrow \infty$.

Case 2: $\left(u_{n}^{1}-u_{n}^{2}\right)$ is nonvanishing. Since $m, m^{-1}$ and $I^{\prime}, \Psi^{\prime}$ are all equivariant with respect to translation of the form $u \mapsto u(\cdot-k)$ with $k \in \mathbb{Z}^{3}$, we may assume that the sequence $\left(y_{n}\right)$ is bounded in $\mathbb{R}^{3}$. Passing to a subsequence once more, there exist $u^{1}, u^{2}$ and $\alpha^{1}, \alpha^{2}$ such that

$$
u_{n}^{1} \rightarrow u^{1}, \quad u_{n}^{2} \rightarrow u^{2}, \quad\left\|u_{n}^{1}\right\| \rightarrow \alpha^{1}, \quad\left\|u_{n}^{2}\right\| \rightarrow \alpha^{2},
$$

and $I^{\prime}\left(u^{1}\right)=I^{\prime}\left(u^{2}\right)=0$. By (3.8) we have that $u^{1} \neq u^{2}$, and it follows from Lemma 3.2(2) that

$$
\sqrt{\alpha_{0}} \leq \alpha^{i} \leq v(d):=\sup \left\{\|u\|: u \in I^{d} \cap M\right\}, \quad i=1,2
$$

(that $v(d)<\infty$ is a consequence of Lemma 3.3(3)).

Suppose $u^{1}, u^{2} \neq 0$, then $u^{1}, u^{2} \in M$. We put $v^{1}:=m^{-1}\left(u^{1}\right)$ and $v^{2}:=m^{-1}\left(u^{2}\right)$. Then $v^{1}, v^{2} \in H$ and we have

$$
\liminf _{n \rightarrow \infty}\left\|v^{1}-v^{2}\right\|=\liminf _{n \rightarrow \infty}\left\|\frac{u^{1}}{\left\|u^{1}\right\|}-\frac{u^{2}}{\left\|u^{2}\right\|}\right\| \geq\left\|\frac{u^{1}}{\alpha^{1}}-\frac{u^{2}}{\alpha^{2}}\right\|=\left\|\beta_{1} v^{1}-\beta_{2} v^{2}\right\|,
$$

where

$$
\beta_{1}:=\frac{\left\|u^{1}\right\|}{\alpha^{1}} \geq \frac{\sqrt{\alpha_{0}}}{v(d)} \quad \text { and } \quad \beta_{2}:=\frac{\left\|u^{2}\right\|}{\alpha^{2}} \geq \frac{\sqrt{\alpha_{0}}}{v(d)} .
$$

Since $\left\|\left|v^{1}\|=\|\right| v^{2}\right\|=1$, it is easy to see from the inequalities above and Lemma 3.8 that

$$
\liminf _{n \rightarrow \infty}\left\|v^{1}-v^{2}\right\| \geq\left\|\beta_{1} v^{1}-\beta_{2} v^{2}\right\| \geq \min \left\{\beta_{1}, \beta_{2}\right\}\left\|v^{1}-v^{2}\right\| \geq \frac{\sqrt{\alpha_{0}} k}{v(d)} .
$$

It remains to consider the case where either $u^{1}=0$ or $u^{2}=0$. If $u^{2}=0$, then $u^{1} \neq 0$ and

$$
\liminf _{n \rightarrow \infty}\left\|v^{1}-v^{2}\right\|=\liminf _{n \rightarrow \infty}\left\|\frac{u^{1}}{\left\|u^{1}\right\|}-\frac{u^{2}}{\left\|u^{2}\right\|}\right\| \geq \frac{u^{1}}{\alpha^{1}} \geq \frac{\sqrt{\alpha_{0}}}{v(d)}
$$

The case $u^{1}=0$ can be treated similarly. The proof is completed.

As in [27], it is well known that $\Psi$ admits a pseudogradient vector field $Q: S \backslash H \rightarrow T S$ (see [26, Lemma II 3.9]). Let $\eta: \mathcal{G} \rightarrow S \backslash H$ be the corresponding flow defined by

$$
\left\{\begin{array}{l}
\frac{d}{d t} \eta(t, w)=-Q(\eta(t, w)), \\
\eta(0, w)=w
\end{array}\right.
$$

where

$$
\mathcal{G}:=\left\{(t, w): w \in S \backslash H, T^{-}(w)<t<T^{+}(w)\right\} \subset \mathbb{R} \times(S \backslash H)
$$


and $T^{-}(w)<0, T^{+}(w)>0$ are the maximal existence times for the trajectory $t \rightarrow \eta(t, w)$ in negative and positive direction. Note that $\eta$ is odd in $w$ because $Q$ is, and $t \rightarrow \Psi(\eta(t, w))$ is strictly decreasing by the properties of a pseudogradient.

Let $P \subset S, \delta>0$, and define

$$
U_{\delta}(P):=\{w \in S: \operatorname{dist}(w, P)<\delta\}
$$

Below we summarize the properties of $\Psi$ and $\eta$ which will be needed in the proof of Theorem 1.2.

Lemma 3.11 Let $d \geq c$, then for every $\delta>0$ there exists $\varepsilon=\varepsilon(\delta)>0$ such that

(1) $\Psi_{d-\varepsilon}^{d+\varepsilon}=H_{d}$ and

(2) $\lim _{t \rightarrow T^{+}(w)} \Psi(\eta(t, w))<d-\varepsilon$ for $w \in \Psi^{d+\varepsilon} \backslash U_{\delta}\left(H_{d}\right)$.

Proof The claim (1) is an immediate consequence of (3.6) for $\varepsilon>0$ small enough. Claim (2) has been proved in [27], see Lemmas 2.15 and 2.16 there. The argument is exactly the same except that $S^{+}$should be replaced with $S$.

We shall need the notion of genus (c.f. [26]). Set

$$
\Sigma:=\{A \subset S: A \text { is closed and } A=-A\}
$$

Let $\gamma(A)$ denote the usual Krasnoselskii genus of $A$ with the definition that the smallest integer $k$ such that there exists an odd continuous mapping $\sigma: A \rightarrow \mathbb{R}^{k} \backslash\{0\}$. If there is no such mapping for any $k$, then $\gamma(A):=+\infty$. Moreover, $\gamma(\emptyset)=0$.

Proof of Theorem 1.2 (Multiplicity) We consider the nondecreasing sequence of Lusternik-Schinireman values for $\Psi$ defined by

$$
c_{k}:=\inf \left\{d \in \mathbb{R}: \gamma\left(\Psi^{d}\right) \geq k\right\}, \quad k \in \mathbb{N} .
$$

As in [26], we claim that

$$
H_{c_{k}} \neq \emptyset \quad \text { and } \quad c_{k}<c_{k+1} \quad \text { for all } k \in \mathbb{N} \text {. }
$$

If this is true, then it follows that there exists an infinite sequence $\left( \pm w_{k}\right)$ of pairs of geometrically distinct critical points of $\Psi$ with $\Psi\left(w_{k}\right)=c_{k}$, contrary to (3.6), and our proof is completed.

To prove (3.9), let $k \in \mathbb{N}$ and put $d=c_{k}$. From Lemma 3.8, $H_{d}$ is either empty or a discrete set, hence $\gamma\left(K_{d}\right)=0$ or 1 . By the continuity property of the genus, there exists $0<\delta<\frac{k}{2}$ such that $\gamma(\bar{U})=\gamma\left(K_{d}\right)$, where $U:=U_{\delta}\left(K_{d}\right)$. For such $\delta$, choose $\varepsilon>0$ such that the conclusions of Lemma 3.11 hold. Then for each $w \in \Psi^{d+\varepsilon} \backslash U$ there exists $t \in\left[0, T^{+}(w)\right)$ with $\Psi(\eta(t, w))<d-\varepsilon$, so we may denote the smallest time for which $\Psi(\eta(t, w)) \leq d-\varepsilon$ by $e(w)$. Since $d-\varepsilon$ is not a critical value of $\Psi$ by Lemma 3.11, it is easy to see by the implicit function theorem that $e$ is a continuous mapping and, since $\Psi$ is even, $e(-w)=e(w)$ (see Lemma 3.5(4)). As a consequence, the mapping $h: \Psi^{d+\varepsilon} \backslash U \rightarrow \Psi^{d-\varepsilon}$ satisfying 
$h(w)=\eta(e(w), w)$ is odd and continuous, so it follows from the properties of the genus and the definition of $c_{k}$ that $\gamma\left(\Psi^{d+\varepsilon} \backslash U\right) \leq \gamma\left(\Psi^{d-\varepsilon}\right) \leq k-1$, which implies

$$
\gamma\left(\Psi^{d+\varepsilon}\right) \leq \gamma(\bar{U})+k-1=\gamma\left(K_{d}\right)+k-1 .
$$

If $\gamma\left(K_{d}\right)=0$, then from above we have $\gamma\left(\Psi^{d+\varepsilon}\right) \leq k-1$, contrary to the definition of $c_{k}$. Therefore, $\gamma\left(K_{d}\right)=1$ and so $K_{d} \neq \emptyset$. Suppose $c_{k+1}=c_{k}$, then by (3.10), the definition of $d=c_{k}$ and of $c_{k+1}$ we deduce that $\gamma\left(K_{d}\right) \geq 2$, contrary to the fact that $\gamma\left(K_{d}\right)=1$. Hence $c_{k}<c_{k+1}$ and so (3.9) is obtained. The proof is completed.

\section{Acknowledgements}

The authors would like to thank the anonymous referees for their valuable suggestions and comments.

\section{Funding}

J. Zhang was supported by the Natural Science Foundation of Inner Mongolia Autonomous Region (No. 2019MS01004 2019LH01002) and the National Natural Science Foundation of China (No. 11962025).

\section{Availability of data and materials}

We declare that materials described in the manuscript are freely available to any scientist wishing to use them for noncommercial purposes, without breaching participant confidentiality.

\section{Competing interests}

The author declares that they have no competing interests.

\section{Authors' contributions}

The authors declare that the study was realized in collaboration with the same responsibility. All authors read and approved the final manuscript.

\section{Publisher's Note}

Springer Nature remains neutral with regard to jurisdictional claims in published maps and institutional affiliations.

Received: 16 April 2021 Accepted: 23 August 2021 Published online: 15 September 2021

\section{References}

1. Alves, C.O., do Ó, J.M., Miyagaki, O.H.: On nonlinear perturbations of a periodic in $\mathbb{R}^{2}$ involving critical growth. Nonlinear Anal. 56, 781-791 (2004)

2. Alves, C.O., Souto, M.A.S., Soares, S.H.M.: Schrödinger-Poisson equations without Ambrosetti-Rabinowitz condition. J. Math. Anal. Appl. 377, 584-592 (2011)

3. Azzollini, A., Pomponio, A.: Ground state solutions for the nonlinear Schrödinger-Maxwell equations. J. Math. Anal. Appl. 345, 90-108 (2008)

4. Benmilh, K., Kavian, O.: Existence and asymptotic behaviour of standing waves for quasilinear Schrödinger-Poisson systems in $\mathbb{R}^{3}$. Ann. Inst. Henri Poincaré, Anal. Non Linéaire 25, 449-470 (2008)

5. Berestycki, H., Lions, P.L.: Nonlinear scalar field equations, l: existence of a ground state. Arch. Ration. Mech. Anal. 82, 313-346 (1982)

6. Cerami, G., Vaira, G.: Positive solutions for some non-autonomous Schrödinger-Poisson systems. J. Differ. Equ. 248 $521-543(2010)$

7. Chen, L.Z., Feng, X.J., Hao, X.N.: The existence of sign-changing solution for a class of quasilinear Schrödinger-Poisson systems via perturbation method. Bound. Value Probl. 2019, 159 (2019)

8. Colin, G.M.: A multiplicity result for nonlinear Schrödinger-Maxwell equation. Commun. Appl. Anal. 7, 417-423 (2003)

9. Colin, G.M., Jeanjean, L.: Solutions for a quasilinear Schrödinger equation: a dual approach. Nonlinear Anal. 56, 213-226 (2004)

10. D'Ambrosio, L., Mitidieri, E.: Quasilinear elliptic systems in divergence form associated to general nonlinearities. Adv. Nonlinear Anal. 7, 425-447 (2018)

11. D’Aprile, T., Mugnai, D.: Solitary waves for nonlinear Klein-Gordon-Maxwell and Schrödinger-Maxwell equations. Proc. R. Soc. Edinb., Sect. A 134, 893-906 (2004)

12. D'Aprile, T., Wei, J.C.: On bound states concentrating on sphere for the Maxwell-Schrödinger equations. SIAM J. Math. Anal. 15, 321-342 (2005)

13. Ding, L., Lin, L., Meng, Y.J., Zhuang, G.L.: Existence and asymptotic behavior of ground state for quasilinear Schrödinger-Poisson system in $\mathbb{R}^{3}$. Topol. Methods Nonlinear Anal. 47, 241-264 (2016)

14. do Ó, J.M., Severo, U.B.: Quasilinear Schrödinger equations involving concave and convex nonlinearities. Commun. Pure Appl. Anal. 8, 621-644 (2009)

15. Fang, X.D., Szulkin, A.: Multiple solutions for a quasilinear Schrödinger equation. J. Differ. Equ. 254, 2015-2032 (2013)

16. Figueiredo, G.M., Siciliano, G.: Existence and asymptotic behaviour of solutions for a quasi-linear Schrödinger-Poisson system under a critical nonlinearity (2017) arXiv:1707.05353 
17. Figueiredo, G.M., Siciliano, G.: Quasi-linear Schrödinger-Poisson system under an exponential critical nonlinearity: existence and asymptotic behaviour of solutions. Arch. Math. 112, 313-327 (2019)

18. Ianni, I.: Solutions of the Schrödinger-Poisson problem concentrating on spheres, part II: existence. Math. Models Methods Appl. Sci. 19, 877-910 (2009)

19. Ianni, I., Varia, G.: Solutions of the Schrödinger-Poisson problem concentrating on spheres, part I: necessary condition. Math. Models Methods Appl. Sci. 19, 707-720 (2009)

20. Illner, R., Kavian, O., Lange, H.: Stationary solutions of quasi-linear Schrödinger-Poisson system. J. Differ. Equ. 145, $1-16(1998)$

21. Ji, C., Fang, F., Zhang, B.L.: Least energy sign-changing solutions for the nonlinear Schrödinger-Poisson system. Electron. J. Differ. Equ. 2017, 282 (2017)

22. Li, W., Rădulescu, V., Zhang, B.L.: Infinitely many solutions for fractional Kirchhoff-Schrödinger-Poisson systems. J. Math. Phys. 60, 011506 (2019)

23. Liu, J.Q., Wang, L.Q., Wang, Z.-Q.: Soliton solutions for quasilinear Schrödinger equations II. J. Differ. Equ. 187, 473-493 (2003)

24. Mao, A.M., Chang, H.J.: Schrödinger-Poisson system with radial potentials and discontinuous quasilinear nonlinearity. Topol. Methods Nonlinear Anal. 51, 79-89 (2018)

25. Shen, L.J: Ground state solutions for a class of generalized quasilinear Schrödinger-Poisson systems. Bound. Value Probl. 2018, 44 (2018)

26. Struwe, M.: Variational Methods, Applications to Nonlinear Partial Differential Equations and Hamiltonian Systems, 3rd edn. Springer, Berlin (2000)

27. Szulkin, A., Weth, T.: Ground state solutions for some indefinite variational problems. J. Funct. Anal. 257, 3802-3822 (2009)

28. Szulkin, A., Weth, T.: The method of Nehari manifold. In: Gao, D.Y., Motreanu, D. (eds.) Handbook of Nonconvex Analysis and Applications, pp. 597-632. International Press, Boston (2010)

29. Willem, M.: Minimax Theorems. Birkhäuser, Boston (1996)

30. Xue, Y.F., Tang, C.L.: Existence of a bound state solution for quasilinear Schrödinger equations. Adv. Nonlinear Anal. 8, 323-338 (2019)

31. Ye, Y.W., Tang, C.L.: Existence and multiplicity of solutions for Schrödinger-Poisson equations with sign-changing potential. Calc. Var. Partial Differ. Equ. 53, 383-411 (2015)

32. Yin, R., Zhang, J., Shang, X.: Positive ground state solutions for Schrödinger-Poisson system with critical nonlocal term in $\mathbb{R}^{3}$. Math. Methods Appl. Sci. 43, 8736-8752 (2020)

33. Zhang, H., Xu, J.X., Zhang, F.B.: Positive ground states for asymptotically periodic Schrödinger-Poisson systems. Math. Methods Appl. Sci. 36, 427-439 (2013)

34. Zhang, H., Xu, J.X., Zhang, F.B., Du, M.: Ground states for asymptotically periodic Schrödinger-Poisson systems with critical growth. Cent. Eur. J. Math. 12, 1484-1499 (2014)

35. Zhang, J., Guo, L., Yang, M.: Quasilinear asymptotically periodic Schrödinger-Poisson system with subcritical growth. Bound. Value Probl. 2020, 109 (2020)

36. Zhao, L., Liu, H.D., Zhao, F.K.: Existence and concentration of solutions for the Schrödinger-Poisson equations with steep well potential. J. Differ. Equ. 255, 1-23 (2013)

\section{Submit your manuscript to a SpringerOpen ${ }^{\circ}$ journal and benefit from:}

- Convenient online submission

- Rigorous peer review

- Open access: articles freely available online

- High visibility within the field

- Retaining the copyright to your article

Submit your next manuscript at $\gg$ springeropen.com 